István BERSZÁN

Faculty of Letters, Babeș -Bolyai University

Cluj-Napoca, Romania

berszan.istvan@ubbcluj.ro

\title{
TIME AS THE RHYTHM OF THEORETICAL PRACTICES
}

Recommended Citation: Berszán, István. "Time as the Rhythm of Theoretical Practices”. Metacritic Journal for Comparative Studies and Theory, 7.2 (2021). Doi: https://doi.org/10.24193/mjcst.2021.12.12

Abstract: Many scholars would agree today that no theory is timeless. But they would probably mean the historicity of theoretical thinking, including the concepts and preconceptions of time propagated by theoretical literary studies. In this paper, I will investigate a usually ignored but unavoidable problem: the rhythmic dimension of theorising. How do those practices, to which different theoretical attempts are linked, influence their orientation in time(s)? Gathering positive data of the past in positivism, revealing the work of formal devices of poetic language in formalism, following rhetorical (ex)changes in postmodernism or reducing every kind of change to historical construction are not only ideological patterns but practical rhythms considered as paradigmatic for other - and sometimes for all - happenings. Based on practiceoriented physics, I propose research of time projections by which literary reading and writing are transposed to the kinetic spaces of certain theoretical practices.

Keywords: theoretical practice, rhythm, kinetic space, time projection, literary reading and writing

My paper investigates how theories deal with multiple, disjunctive, incommensurable experiences of time including literary writing and reading. Experiencing time has to do with a universe or multiverse of rhythms: natural phenomena of very different kinds; a proliferation of inner happenings; uncountable individual and social practices; incommensurable cultural rituals; ways of historical memory; timetables, programs, 185 
deadlines; scientific discoveries of previously unknown processes etc. This heterogeneity is explained by physical laws, geographic locality, climate (change), evolution, historical contexts, cultural differences, scientific progress or other macro-level "frames" of time. In order to approach disjunctive temporalities, I have proposed a practice-oriented physics in which I have introduced the notion of complementary rhythmic dimension as an extension of the string theory to time directions of practices (Berszán).

The geometry of complementary curled up space dimensions in string theory preserves the single extended time dimension - however, taking into consideration that time is not only measurable duration but rhythm as well, besides its passing, we also have to deal with different rhythmic directions. This time, direction ceases to stretch along a straight line, it becomes the rhythm of a movement and, consequently, changing direction in time is equal to changing the rhythm. I consider approaches to a happening from different disciplinary or theoretical directions as time projections of that happening according to the complementary rhythmical dimension of the applied research practice. Every research practice has its special complementary rhythmic dimension - deconstruction, for instance, is happening in the rhythmic dimension of rhetoric change, we enter its kinetic space by our attentive gestures tuned to the play of signifiers.

The main question in this paper is how theories like structuralism or deconstruction succeed in encompassing diverse and heterogeneous rhythms of happening in their paradigmatic kinetic space of theorising? From Russian formalists to postmodernist criticism, literary theories revealed basic principles or movements (such as formal devices of poetic language or the uncontrollable rhetoricity of discourse) that seemed to be general and historically speaking "timeless". Following them, they tried to deduce and explain what happens in literature. We all agree today that no theory is timeless. But what means the time of a theory? We can consider the historical contexts in which it was elaborated; we can consider the concepts and preconceptions of time used or involved by it. But theory always has its rhythm as well; I mean the practical rhythm we are tuned to by theorising in a way or another. Traditionally we oppose theory with practice, but, after all, theorising is itself a certain practice - of course, not always the same. 
How the rhythmical dimension of theorising is relevant concerning our orientation in time(s) and in encompassing disjunctive temporalities? We already said that theories are attempts to find out what is happening in certain phenomena in order to understand and explain them. How do they find that happening? Let's begin with history. After the choices of Enlightenment, theory tries to grasp immanent connections that form working wholes. Natural sciences described physical and biological systems that explain the movement of planets or the evolution of species. Some researchers were curious whether systemic orientation can be reliable in society and cultural practices as well. By these attempts modern social and human sciences offered an immanent orientation in our world's phenomena, but ignored diverse temporalities. Modelling became the basic method of explaining different phenomena by reducing them to the same) simplified mechanisms and selected principles. Especially theory became a way of universal orientation in all sorts of happenings that had been (sharply) separated before (take literature and fashion, for instance). But even a "universal orientation" in time(s) has its own rhythm: it is necessarily tuned to the temporality of a chosen motion and/or practice.

Some theories orientate themselves in new areas according to an already known way of happening (structuralism follows system functioning in society, language, literature and culture) - other theories discover a new kind of occurrence by which they modify orientation in already investigated areas (deconstruction discovers the uncontrollable play of signifiers, and provides a sheer criticism of the centre-based knowledge of Western philosophy and Western thinking in general). Time is motion, so it has different rhythms according to what is moving. Structuralists and deconstructors are not searching for moral examples in literature, nor gathering documents of a historical period, but reveal textual constructions or follow textual activity. Orientation in time does not depend only on concepts and preconceptions of time but on practical rhythms as well. "System" is a theoretical concept but it is linked to a structuralist practical orientation searching for functional connections. Even though such attempts can be related to historical processes, they have their own complementary rhythmic dimensions. Surely, it is historically determined when theory operates with working 
systems, but at the same time, the history of theory will depend for a long time on the research of system functioning.

The rhythm of structuralism is adapted to the repeating functional levels of a system. Roman Ingarden, for instance, proposes a phenomenological layered structure of the literary work, creating the functional unity of five relatively independent strata: sound, meaning, schematic images, represented objects and metaphysical qualities (Ingarden, The Literary). Barthes offered a layered semiotic system of culture that includes fashion and politics beside natural language. Thus, langue as the final or central system of forms functionally integrates different realms of human life. How is it possible to encompass heterogeneous rhythms? Such an undertaking needs two practical skills: 1 . orientation in time tuned this time to functional inclusion into a system. In this kinetic space typical events are the ways how components get functions in a larger whole; 2. the second practical skill is the theoretical elaboration of precise projections of investigated phenomena and/or practices to the rhythmic dimension of system functioning. Modelling as simplifying complex or diverse occurrences by reducing them to certain mechanisms and principles is equal to their time projection.

In his 1964 study, Elements of Semiology, Roland Barthes writes:

The aim of semiological research is to reconstitute the functioning of the system of signification other than language in accordance with the process typical of any structuralist activity, which is to build a simulacrum of the objects under observation (Barthes 1968, 95).

This means that different systems can never deviate from operating as signifying systems. This is not to say that nothing else happens in the linguistic and non-linguistic phenomena in question, but it is through their structuralist reconstruction that they become signifying systems. Barthes relies on the principle of relevance of structuralism, which focuses on the meaning of the objects analysed, i.e., it limits its interest to the process of meaning construction. He notes that other kinds of relevance should not be denied; however, methodologically consistently, he immediately adds that those, too, need to be discussed in semiological terms, that is, their place and function should be designated in the system of meaning. The semiologist is not interested in either the 
economics or the system of fashion, they only point out at which level of the semiotic system of fashion are economics and sociology related to semiological relevance. And the same is true as regards the study of the French alimentary system: in this approach, it must also function as a signifying system.

Relevance is nothing else than laying down methodologically the rhythm/practice one chooses to follow, and it is closely related to the selected, more exactly the homogenised corpus of documents. Barthes emphasises the homogeneity of substance and the homogeneity of temporality. An example of the homogeneity/homogeneous nature of substance is linguistics, which works only with phonic substance. In the French alimentary system, this would correspond to working with only one type of document (e.g. restaurant menus). When the homogeneity of the corpus cannot be maintained (e.g. clothing and written language in fashion, or image, music and speech in cinema), Barthes suggests the same strategy as in the case of alternative relevance: to concentrate on the systematic structuring of the substances used, that is, their heterogeneity itself is interpreted structurally, and thus all kinds of heterogeneity are homogenised as subsystems in the same comprehensive signifying system developed for the modelling of language.

"Further, homogeneous in time" (Barthes 1968, 98). Barthes refers only to the elimination of diachronic elements so that the corpus would coincide with a certain state of the system, a "cross-section of history", but the homogenisation of temporality in the organisation of the corpus follows after something. In other words, the homogeneity of time means not only the synchronical present of the selected corpus elements but also a specific monotemporality: the exclusive operation of the signifying system everywhere.

The goal of all structuralist activity, whether reflexive or poetic, is to reconstruct an 'object in such a way as to manifest thereby the rules of functioning ('functions') of this object. (...) the imitated object makes something to appear, which remained invisible or, if one prefers, unintelligible in the natural object (Barthes 1972, 214-215).

Ultimately, it is the meaning added to the object that manifests itself, the recreated object does not resemble the natural object in order to copy it, but in order to make it intelligible. It is not about its mere symbolic function, but about understanding how it 
works. The structuralist literary scholar, for example, explores not only what the literary text means, but also what happens in literature. And since what happens depends on structuralist activity focused on the study of all kinds of objects, it is no wonder that Barthes affirms something like: "along with economics, linguistics is, in the present state of affairs, the true science of structure" (Barthes 1972, 213).

As a theorist of late structuralism he recognises the relevance of practical orientation in research, however, in spite of the relativisation of his own technique, he retains its invariants as if his insights would not apply to them:

It is therefore to the degree that the goals of structuralist activity are indissolubly linked to a certain technique that structuralism exists in a distinct fashion in relation to other modes of analysis or creation: we recompose the object in order to make certain functions appear, and it is, so to speak, the way that makes the work; this is why we must speak of the structuralist activity rather than the structuralist work (Barthes, Structuralist 216).

This conception of structuralist activity extended to the arts, too, considers the creation of meaning to be more important than the meanings themselves:

[i]t is because the function is extensive with the works that structuralism constitutes itself as an activity, and refers the exercise of the work and the work itself to a single identity: a serial composition or analysis by Lévi-Strauss are not objects, except insofar as they have been made: their present being is their past act: they are having-beenmades (Barthes 1972, 219).

Practice-oriented physics focuses on the rhythm of this activity or practice and examines how this activity or practice reflects the time projections of other rhythms and practices.

Still, this activity that excludes diachrony considers history important in one single respect: as the story of the man creating meaning, but, of course, in a synchronous view "as if it could not be the content of meanings that exhausted the semantic goals of humanity, but only the act by which these meanings, historical and contingent variables, are produces" (Barthes, 1972, 218). Even the thesis of the historical 
emergence and expected disappearance of structuralism remains within the kinetic space of structuralist activity, "structuralism, too, is a certain form of the world, which will change with the world" (Barthes 1972, 219). But the history of the world remains the history of forms, so structuralism can only end this way: after it has told the story of the old languages of the world in a new way, a new language will emerge from history, which tells its story. The reduction of happenings in structuralism means that everything can only happen in the signifying system, including the disappearance of structuralism. From this point of view, Homo significans seems to be not only the new man of structural research but also the invariant of (structuralist) history.

Elements of Semiology ends with these words: "it is impossible to guess the speed at which systems will alter, since the essential aim of semiological research (that is, what will be found last of all) may be precisely to discover the systems own particular time, the history of forms" (Barthes 1968, 98). The systems of structuralism have their own particular time and history - the time and history of meaning creation that coincides with structuralist activity not only by definition but also in the time projection of all examined happenings on the rhythmic dimension of structuralist activity. In this way, however, one loses sight of the "own particular time" of events outside the signifying system, we fail to follow them in the rhythmic dimensions in which they take place. The methodological principle of relevance creates homogeneity in substance and temporality not only by selecting certain defining properties of the object but also by assigning the objects to the functions of the signifying system operated by structuralist activity. This is done not so much through the functions projected onto them, but rather through the systematisation of the objects created as projections. Barthes emphasises that the object is reconstructed by structuralist activity in accordance with its own relevance principle; in other words, it is transformed into an element or structure of the signifying system. The application of the principle of relevance means from the outset the selection of objects as homogeneous substances of the relevant activities. Just as their temporal homogeneity is created by their involvement in the monotemporal synchronicity of the relevant functions. Above all, structuralism "manifests a new category of the object, which is neither the real nor the rational, but the functional" (Barthes 1972, 218). Thus, one does not extract from the object what one is looking for 
but rather shapes the object so that one can study in it what all structuralist inquiries are looking for.

Barthes specifies the operations of structuralist activity as fragmentation according to linguistic paradigms and arrangement. I would rather show (in these as well) that they transform objects into apertures that make only system functions visible. This brings about homogenisation: through time projections, every object and every corpus changes into an aperture that opens up to system functions. But this gap is not only a frame or the lack of something, but the opening up (or aperture) of a kinetic space, through which I can only enter that specific kinetic space, and practically my attention can be focused only on the gestures made within that space. There is no opening in the wall of the cinema, through which we can see the events of the film world. Instead, the film directs our gaze to such an opening created by projection so that we could see exactly what, or only what unfolds throughout that opening. Just as the film spreads over the wall, the structuralist projection of the object also covers the "natural" object. The wall should not be painted in strong colours, it should be made uniformly white so as it would not disturb what is seen "through it". However, we can't see through the wall, in fact, we see something on the wall instead of the wall. Time projection, too, allows us to see everything that happens (there) as happening in a single space. The opening is not a mere lack of something, but a kinetic space chosen through practice, this time through structuralist activity. Therefore, our option is never abstract selection, but a practical change of rhythm: to drive our otherwise diverse viewing practices into the kinetic space of the gestures of theorising, on the one hand through the strong impulses of theoretical inquiry, and on the other through our attempts at attunement with these.

The time projections of structuralist activity can be followed by us only if we are involved in shaping them; if they become given through the attention practices of our watching, reading or contemplation. In other words, we have to turn the object transformed into some kind of opening into our eyeslits to see what can be seen in it. As soon as we are cajoled into such gestures and practices of attention, we become a screen in the opening of which the history of structuralist "forms" (projections) takes place. The "own time" of system operation thus coincides with our actual structuralist kinetic 
space, and what we perceive in the meantime counts as real (or the only reality) because we perceive it through our ongoing gestures. The real counterpart of a time projection is our temporal orientation determined by our gestures.

The thing that the structuralist theorist and the artist have in common is that they both try to cajole us into orienting ourselves in time. Yet, they drive us into completely different kinetic spaces, which we cannot grasp at once in some neutral space because there is no such neutral space. Whatever we do, what happens to us always happens in a specific kinetic space. Practical orientation in time means that I either stay in one kinetic space or I step out of it so that I tune myself to the happenings of another kinetic space. While art undertakes to search for different kinetic spaces, theory is characterised by being strictly "consistent", which means that, apart from its demanding scientific standards, it is also unwilling to step out of its own paradigmatic kinetic space. This can only happen through the creation of another theory. It is due to this fact that structuralism has remained structuralism up until this day, but Roland Barthes steps out of its kinetic space as a theorist in $S / Z$, which necessarily coincides with his break with structuralism. Poststructuralism is not only a historical change in the sense of temporal succession or consequence/development, but inevitably a change in the rhythm of practical orientation in time, too.

Deconstruction has drastically changed the rhythm of structuralist orientation in time - system construction is projected to the rhythmic dimension of a more dynamic happening: the play of signifiers. Text is no more a result of transformations controlled by invariant principles, but an activity continually deconstructing itself, which cannot be reduced to something more idiomorphic. The formation, transformation and dislocation of the system are prior to the static, hierarchic connections of its elements around a centre. Thus, theory provides orientation in the kinetic space of rhetoric happening and concepts like change, play, movement are elaborated according to this temporality.

By struggling against the the(rr)oretics of the centred structure and reasserting the "rights" of history put into brackets in structuralism, Derrida shepherds theorising towards a rhythm tuned to the structurality of structure. Such dislocation of structuralist activity transforms the object of deconstruction as well, but in a different way than structuralist activity did: the proposed technique of double reading elaborates time projections cast on the kinetic space of its own practical orientation. The events we 193 
can follow in this case are rupture and redoubling, these constitute the rhythm of deconstruction. Instead of a centred structure, Derrida reveals a playfield and consequently motion, change, and temporality everywhere. But, as the structuralist activity obscured the "natural" object, the attempt of projecting motion, change, play and temporality to the kinetic space of deconstructive double reading also obscures alternative rhythms of motion, change, play and temporality.

In his programmatic paper, entitled Structure, Sign and Play in the Discourse of Human Sciences, Jacques Derrida speaks about a turn that deprives the inflexible structure of its centre and liberates it as a space of the unpredictable, i.e. event-like play. The first paragraph speaks about this prototypic event: "Perhaps something has occurred in the history of the concept of structure that could be called an 'event'. (...) Its exterior form would be that of a rupture and a redoubling" (Derrida 351). As we follow the steps of his critical argumentation it seems, indeed, that this turn affects the whole history of Western thinking producing fault-lines everywhere and in everything we imagined before as a whole. The guiding centre is unmasked as something that limits the play of structure: the unpredictable combination of elements is narrowed and closed by an organising principle. On the other hand, the centre becomes suspicious because we cannot localise it either inside or outside the structure - it withdraws from the play of structure by being located outside of itself as the presence of an invariant but, in fact, always substituted by its signifiers: "Successively, and in a regulated fashion, the centre receives different forms or names. The history of metaphysics, like the history of the West, is the history of these metaphors and metonymies" (Derrida 353). These are, in fact, the objects of the desire for final knowledge.

If this is the case, then the critique of the centred structure is justified, but as a result of decentralisation everything occurs in the system of discourse as a system of signifying differences: "The absence of the transcendental signified extends the domain and the play of signification infinitely" (Derrida 354). This "turn" goes beyond the attempts of the "destructors" (Nietzsche, Freud and Heidegger), it coincides with the rupture identified as the prototype event. Similarly, as the continuous operation of the structurality of structure has been neutralised by a process or gesture that gave it a centre, play and motion are reduced to the play of signifiers by the deconstructive gesture projected on them, or rather by their projection onto the gestures of 
deconstruction. The gesture of deconstruction reads simultaneously the hierarchical oppositions of the system and the uncontrollable play of signifiers obscured, surpassed or marginalised by those oppositions. But we have to examine what kind of play is revealed by deconstruction.

The problem with the attempts of destructors is that they get stuck in metaphysics because they destroy a centralised system by creating a new one. In contrast to them, deconstruction uses the concepts of the criticised system, showing on the inside how the system deconstructs itself. Thus, we escape to freeze our reading as a new metaphysical system, but we reveal the play - just liberated from the tyranny of the centred organising principle - exclusively as the play of signifiers. In other words, deconstruction itself is "stuck" in the rhythm of the discourse. Lévi-Strauss' structuralist ethnology is an opportune medium for studying the discursive aspect of play, however, by considering its rhythmic dimension as paradigmatic for other occurrences, we obscure the non-discursive practices of play in a similar manner as the centre has obscured the structurality of the structure. Whether we speak about the opposition between nature and culture, about bricolage or the similarity between myths and LéviStrauss' investigations, the only relevant features are the critique of traditional language, the exclusively conceptual tools of bricolage and the mythologic or mythomorphic discourse of mythopoetics. As if the relation between nature and culture would be exclusively the part of a conceptual system, as if the multiple skills of bricolage could be reduced to the use of concepts or archaic (and contemporary) mythopoetic practices would consist of discourse alone.

According to the nature-culture opposition, everything that is universal and spontaneous belongs to nature, without depending on the norms of any culture; and everything that belongs to culture depends on cultural norms and is transformed by the changing historical or geographical social structures. But Lévi-Strauss finds that the incest prohibition claims the attributes of both nature and culture at the same time because, on the one hand, it is universal (you can find it in every culture), so we should consider it natural, but on the other hand it is a prohibition, i.e. part of a system of norms and consequently must be cultural. I wonder whether the epistemological scandal of incest prohibition has implications only as regards the critique of language or it raises the problem of totalisation of the discourse as well. Derrida only reflects on the first 
because the second one seems to be natural for him - this time not due to the conceptual opposition between nature and culture but by the universalised system of discourse.

We consider natural what goes without saying and is considered an unchangeable system of reference. Since the shadows on the wall of the cave are considered by Plato's prisoners as real, in fact, the only reality, every occurrence is explained according to the nature of these phenomena (see Plato, Republic). Mutatis mutandis, this is valid for the deconstructive time projections cast onto the discursive motion: if every natural and cultural event is projected before us as the play of signifiers, then the signifying system becomes the most natural because everything depends on its play, including conceptual oppositions. Of course, research practice is also synchronised with this play: "Now, ethnology - like any science - comes about within the element of discourse" (Derrida 356). Neither fieldwork nor experiments are considered exceptions because we follow them too "within the element" of discourse. Just as in a physics tailored on light propagation we cannot suppose a higher speed than the speed of light, in the human sciences tailored on the play of signifiers nothing can move, but symbols, concepts or other tropes. It doesn't matter what we examine because orientating ourselves in the system of signifiers we match everything to its functioning, including, of course, our methodology. Everything that could be supposed beyond the system of signifying differences will be considered as myth or impossible.

Even bricolage must be reduced to discourse: "If one calls bricolage the necessity of borrowing one's concepts from the text of a heritage which is more or less coherent or ruined, it must be said that every discourse is bricoleur" (Derrida 360). Or again: "There is, therefore, a critique of language in the form of bricolage, and it has even been said that bricolage is critical language itself" (Derrida 360). If anything happens to point outside of this system - an engineer, for instance, invoked by Lévi-Strauss as the other of the bricoleur, who eventually can do more or something else than just speaking -, it must be interpreted as a mythopoetic function of bricolage. Derrida demonstrates that "the notion of the engineer who supposedly breaks with all forms of bricolage is therefore a theological idea" (Derrida 360) or, in other words, it has no real foundation. He continues with a gesture that breaks with academic style, suggesting that his 
supposition can be accepted as a fact: "the odds are that the engineer is a myth produced by the bricoleur" (Derrida 360).

How can a scholar afford to wager in an argumentation? This time, not as an illustration of the bricolage but because of sharing a natural system of reference with structuralism: they both accept that everything happens in a linguistic or discursive space. Thus, together with the centre of the structure, deconstruction has to disclaim any playing ground beyond the system of signifiers and any possibility to get beyond the philosophy of discourse: "Transphilosophical concepts would be transformed into philosophical naivety" (Derrida 364). We could label this as the scandal of the obscured temporalities of the occurrences projected onto the discourse. Even though deconstruction wants to rehabilitate play against all static guiding principles, it is equally obvious that from the point of view of this theory every event and play happens in the space of discourse because in the kinetic space of deconstruction this is the only real, ongoing and, in this sense, natural moving. According to Barthes' relevance principle, Lévi-Strauss claims that "the reintegration of culture in nature and finally of life within the whole of its physico-chemical conditions" (Lévi-Strauss 247) is the job of exact natural sciences. Derrida, however, quotes it exclusively from the point of view of the construction and deconstruction of a conceptual opposition between nature and culture (see Derrida 360). Both theory and practice of deconstruction consist of projecting multiple rhythms to the rhythmic dimension of discursive play. This reduction obscures that deconstructing conceptual-rhetorical oppositions is not the only way to get beyond a system; we can step out of its rhythm or play also by changing our practical orientation and tuning our reading or research experiments to the temporalities of other occurrences - for instance, to those gestures of attention that literary writing prompts us by practising them.

Structuralist theorising reduces disjunctive time experiments to functional inclusion controlled by a central system. Deconstructivist theorising seems to discover the real force of time that can never be fully controlled, and the real events dislocating the system itself. But this uncalculatable change is tuned to the play of signifiers in which everything and anybody takes their rhetoric chances. Thus, theory delimits a kinetic space where time is reduced to the paradigmatic pattern of happening, in our case to the functional inclusion or rhetoric change. Such kinetic spaces are singled out 
by orientation practices that make us follow every happening in the same rhythmic dimension. To "understand what happens in literature" is equal to reducing it to the rhythm of the proposed theorising.

Out of scientific exigence, there must be a connection between the rhythm of theorising and the happening of the investigated phenomena. Ideally, they should be the same, but since they are not, the solution is a time projection of the latter to the kinetic space of the first. The results are literary work as system construction or writing and reading as textual activity. In fact, theoretical work often consists of this rhythmic reduction. The theorist is expected to elaborate a model which explains (m)any happenings he/she investigates. This job is not only an interpretation of those phenomena but a time projection of their happening as well. In the Platonic cave of structuralist scholars, we must follow any occurrence in the rhythmic dimension of system functions without turning our attention toward any other rhythms. As a consequence, poems, stories and dramas are converted into special types of texts making it impossible to discover them as different orientations in time(s). In a structuralist approach, there are possible many kinds of transformations inside or between literary works, except changing the rhythm of system functions.

Similarly, but in a different complementary time dimension, deconstructive theorising projects even ethical orientation to the kinetic space of text reading. Ethical obligation appears in Hillis Miller's The ethics of reading as an imperative of deconstruction: the reader should read the text answering the complex of signifiers. Hence, Derrida's warning that text is never a transparent window, and consequently we have to take into account the production of the glass, is validated as the ethics of reading. Robert Eaglestone criticises the reduction of the reader's responsibility to the exigencies of a text (see Eaglestone, Flaws). I would add that deconstruction is closed in the Platonic cave of rhetoric happenings, this is why ethics is narrowed to answering signifiers. Arguing that the narrative or figural exemplification of the universal moral law reintroduces contingency into the categoric, Hillis Miller wants to reinforce universal rhetoricity which is possible only by following every "transcendental" happening (those beyond the play of signifiers) as projected to the play of signifiers.

I teach literary theory as practical exercises tuning our experimental reading to different rhythms of theorising. In this way, we can discover, on the one hand, 
distortions caused by time projections and the artful elaboration of the paradigmatic kinetic space of theories, on the other. It is worth learning the art of deconstruction - it becomes problematic just when we accept it as the Foucauldian positivity level of other practices.

At the end of this argumentation, I will formulate some remarks on recent theories of literature. Contemporary literary studies based on historical criticism take time seriously, but they investigate everything in their extended historical dimension. As much as practising literature needs many different ways of paying attention, the complementary rhythmic dimensions of literary writing and reading are to be of interest; otherwise, we lose the multiple, disjunctive, incommensurable experiences of time they provide. Writing and reading literature is a practical enterprise: in the time of writing and reading we have to learn extremely refined and intensive gestures of attention and link them to each other in practice - this is the only way to get and remain in their rhythm. Investigating literature in historical dimension alone is a time projection of literary practices with multiple rhythmic dimensions to the paradigmatic kinetic space of historical construction.

When is theory? In this approach, it is in the time (or moving) of its paradigmatic shadows. According to Plato, it is impossible to convince the prisoners of the cave about any other reality, except their shadows, until we succeed to turn them away from their two-dimensional paradigmatic wall-space and drive them out from the cave to the sunlight. Okay, this sunlight is Plato's cave within the paradigmatic space of Ideas, but there is one more possibility: not to reveal everything in the same light, but to search for the complementary rhythmic dimension of investigated happenings and practices. We always find ourselves in a certain kinetic space and there is no neutral distance between them, but projecting all happenings and practices to a chosen kinetic space or searching to follow them in their rhythmic dimensions are different orientations in time. Histoire croisée, structural time, deep time, Anthropocene and other similar concepts signal the multiple, disjunctive, incommensurable experiences of time. The problem is, that we want to find them all exclusively in the extended historical dimension of happening. It's time to discover their complementary, curled up rhythmic dimensions and enter their incommensurable temporality. 


\section{References:}

Berszán, István. "Empirical Research and Practice-oriented Physics for the Humanities and Sciences." CLCWeb: Comparative Literature and Culture vol. 18, no. 2, 2016. <https://doi.org/10.7771/1481-4374.2860>

Barthes, Roland. Elements of Semiology. Translated by Annette Lavers and Colin Smith. Hill and Wang, 1968.

---. „Structuralist activity”. In Roland Barthes: Critical Essays. Translated by Richard Howard. Northwestern University Press, 1972, pp. 213-220.

---. S/Z. Translated by Richard Miller. Hill \&Wang, 1975.

Derrida, Jacques. "Structure, Sign and Play in the Discourse of the Human Sciences." In Jacques Derrida. Writing and Difference. Translated by Alan Bass. Routledge, 2001, pp. 351-70.

Eaglestone, Robert. "Flaws: James, Nussbaum, Miller, Levinas”. In Critical Ethics: Text, Theory, and Responsibility. Dominic Rainsford, Tim Woods. Macmillan (eds.), 1999, pp. 77-87.

Hillis Miller, Joseph. The Ethics of Reading. Columbia University Press, 1987.

Lévi-Strauss, Claude. The Savage Mind.George Weidenfeld and Nicolson, The University of Chicago Press, 1966.

Ingarden, Roman: The Literary Work of Art. Investigations on the Borderlines of Ontology, Logic and the Theory of Literature. Translated by G. G. Grabowicz. Northwestern University Press, 1973.

Plato. Republic. Translated by P. Shorey. Random House, 1963. 